\title{
A BOOTSTRAP TEST FOR THE PROBABILITY OF RUIN IN THE COMPOUND POISSON RISK PROCESS
}

BY

\author{
BENJAMIN BAUMGARTNER AND RicCARdo GATTO
}

\begin{abstract}
In this article we propose a bootstrap test for the probability of ruin in the compound Poisson risk process. We adopt the P-value approach, which leads to a more complete assessment of the underlying risk than the probability of ruin alone. We provide second-order accurate $\mathrm{P}$-values for this testing problem and consider both parametric and nonparametric estimators of the individual claim amount distribution. Simulation studies show that the suggested bootstrap P-values are very accurate and outperform their analogues based on the asymptotic normal approximation.
\end{abstract}

\section{KEYWORDS}

Edgeworth expansion, exponential and log-normal claim amounts, normal approximation, P-value, pivotal quantity, resampling, second-order accuracy.

\section{INTRODUCTION}

The risk process is a stochastic model for the variations of an insurer's surplus over time. More precisely, if we denote by $r_{0} \geq 0$ the initial reserve, by $c>0$ the constant premium rate and by $\left\{Z_{t}\right\}_{t \geq 0}$ the compound Poisson process of the aggregate claim amounts, then the classical risk process $\left\{Y_{t}\right\}_{t \geq 0}$ is given by

$$
Y_{t}=r_{0}+c t-Z_{t}
$$

for all $t \geq 0$. Precisely, $Z_{t}=\sum_{i=0}^{N_{t}} X_{i}$ for all $t \geq 0$, where $X_{0} \stackrel{\text { def }}{=} 0$, for convenience, $X_{1}, X_{2}, \ldots>0$ are independent individual claim amounts with common distribution function $F$ and independent of the Poisson process $\left\{N_{t}\right\}_{t \geq 0}$ as well. Let us denote by $\lambda>0$ the intensity of the Poisson process and assume that $\mu \stackrel{\text { def }}{=} \mathbf{E}\left[X_{1}\right] \in(0, \infty)$. We define the relative security loading $\beta$ through $c=$ $(1+\beta) \lambda \mu$. The quantity of interest in this article is the probability of ruin within the infinite time horizon, namely the probability that $\left\{Y_{t}\right\}_{t \geq 0}$ ever falls 
below the zero level. It is a well-known risk measure for an insurance company. By defining the time of ruin as

$$
T= \begin{cases}\inf \left\{t \geq 0: Y_{t}<0\right\}, & \text { if this infimum exists, } \\ \infty, & \text { otherwise }\end{cases}
$$

the probability of ruin is usually expressed as $\psi\left(r_{0}\right)=\mathbf{P}(T<\infty)$. We will later use a functional notation for it, see (3) below. We assume $c>\lambda \mu$, i.e. $\beta>0$, otherwise ruin occurs with probability one. Two general references are Gerber (1979) or Bowers et al. (1997, Chapter 13).

Let us denote by $r$ the adjustment coefficient, i.e. the positive solution in $v$ of $\int_{0}^{\infty} \mathrm{e}^{v x} \mathrm{~d} F(x)=1+c v / \lambda$, and assume it exists. An analytical formula for the probability of ruin is given by

$$
\psi\left(r_{0}\right)=\frac{\mathrm{e}^{-r r_{0}}}{\mathbf{E}\left[\mathrm{e}^{-r Y_{T}} \mid T<\infty\right]},
$$

see Bowers et al. (1997, p. 413). Formula (2) can be obtained in various ways: by martingale theoretic arguments, see Asmussen (2000, p. 24-25), by Lundberg conjugation, see Asmussen (2000, p. 69-71), or by ad hoc argumentations, see Bowers et al. (1997, p. 426-427). With the exception of exponentially distributed claim amounts, this formula is however not suitable for numerical evaluations. Various numerical methods for evaluating the probability of ruin have been proposed. Dufresne and Gerber (1989) propose a recursive algorithm based on a discretization and we will use this method in Section 3, see also the Appendix for a summary of it. Asmussen (1985) suggests the use of stochastic simulation based on importance sampling for computing the probability of ruin with a bounded relative error. The saddlepoint approximation is a method of asymptotic analysis that allows to approximate certain complex integrals with a bounded relative error. It provides another efficient way of computing the probability of ruin, see Barndorff-Nielsen and Schmidli (1995) and Gatto (2008). Another approach proposed by Asmussen and Rolski (1991) is to consider phase-type individual claim amount distributions. Phase-type distributions include finite mixtures of exponential and Gamma distributions, but do not include heavy-tailed distributions, like the log-normal. Nevertheless, any distribution on $\mathbb{R}_{+}$can be approximated with arbitrary accuracy by a phase-type distribution. A phase-type individual claim amount distribution leads to a computable formula for the probability of ruin. However, it should be mentioned that phase-type distributions can easily involve a large number of parameters. Estimating these parameters from observed individual claim amounts can be difficult.

In this article we consider a testing problem instead of the single evaluation of the probability of ruin. This allows to take into account the uncertainty of the data used to estimate the unknown model parameters. Furthermore, we 
suggest the P-value approach to this testing problem, which tells us how distant the probability of ruin is from a specified threshold $\psi_{0}$, and we propose computing the P-value by the bootstrap. The bootstrap test relies on the asymptotic normality shown by Pitts (1994) and on the jackknife estimator of the variance of the bootstrap probability of ruin. From a practical point of view, the insurer can fix a threshold value $\psi_{0} \in(0,1)$ for the probability of ruin, above which the business is considered too risky. Because typical probabilities of ruin in both life and non-life sectors are below $1 \%$, the values of the threshold $\psi_{0}$ should also be taken around 1\%. Alternatively, this threshold value could also be imposed by law. We can note, however, that there are presumably insurance companies working under substantially higher probabilities of ruin than $1 \%$. This is often a consequence of insufficient legal constraints, which give important freedom to the companies in building their reserves and thus in offering attractive premiums. The fact that some Swiss health insurance companies have gone to ruin in these recent years seems to confirm this presumption.

More precisely, we propose a bootstrap P-value for the testing problem given by (5) below as a measure of risk. There are obviously alternative measures of risk that are used by insurance companies. Though, the scope of this paper is not to review or to compare various measures of risk, but to propose a new testing problem approach together with an accurate computational method as an improvement to the simple evaluation of the probability of ruin, which is a classical and established risk measure in the actuarial literature.

The rest of this article is organized as follows. At the beginning of Section 2, we give a short review of applications and developments of the bootstrap within the actuarial risk theory. In Subsection 2.1 we provide a precise description of the testing problem proposed, in Subsection 2.2 we give a bootstrap P-value and we justify its asymptotic second-order accuracy. Some concluding remarks are given in Subsection 2.3. In Section 3 we show the numerical accuracy of the proposed P-value by an involved two-level simulation study, in which we consider the exponential and the log-normal claim amount distributions. We also compare our bootstrap P-value with the one obtained from the asymptotic normal approximation and show that the bootstrap P-value is substantially more accurate than the asymptotic normal one.

\section{THE BOOTSTRAP TEST}

The bootstrap goes back to Efron (1979) and some general references are Efron (1982), Hall (1992) and Davison and Hinkley (2003). Some important applications and theoretical developments of the bootstrap in the context of actuarial risk theory are the following. Embrechts and Mikosch (1991) propose a bootstrap procedure for estimating the adjustment coefficient of a risk process under the assumption that the distribution of the number of summands is known, but the common distribution function $F$ of the summands is unknown 
and estimated by a sample. The adjustment coefficient is a central quantity that allows to compute the probability of ruin or the Cramér-Lundberg approximation to it and it is also a measure of risk itself. Their emphasis is on a theoretical justification of the strong consistency of the bootstrap estimator of the adjustment coefficient. Pitts et al. (1996) consider again the case where $F$ is unknown. They propose bootstrap and jackknife confidence bounds for the adjustment coefficient, which is re-expressed as a functional of $F$. Hipp (1989a) considers asymptotic normal and bootstrap confidence intervals for the infinite horizon probability of ruin when $F$ is unknown and a sample from it is available. Pitts (1994) proposes a bootstrap estimator of the distribution function of a random sum, under the assumption that the distribution of the number of summands is known, but the common distribution of the independent summands is unknown and estimated by a sample. The distribution function of the random sum is expressed as a functional of the unknown distribution function of the summands and it is shown that the bootstrap version of this functional is strongly consistent and asymptotically normal, under some continuity and differentiability assumptions on the functional. Bootstrap confidence bands for the whole distribution function are also developed. These results are then applied to the infinite horizon probability of ruin of the classical risk process by using its well-known geometric sum representation, see Pitts (1994, Section 5.4). Politis (2003) considers the classical risk process in the case where both the individual claim amount distribution function $F$ and the Poisson intensity $\lambda$ are unknown. He proves the strong consistency and the asymptotic normality of the bootstrap estimator of the infinite horizon probability of ruin, under general conditions on $F$ and using the functional approach. Loisel et al. (2008) suggest to re-express the finite horizon probability of ruin as a functional of the individual claim amount distribution function $F$ and to use the quantiles of the bootstrap estimator of the finite horizon probability of ruin as a measure of risk. They also provide some formulae for the influence function (which is a directional derivative at $F$ ) of the finite horizon probability of ruin. From this influence function, they obtain the asymptotic variance of the bootstrap estimator of the finite horizon probability of ruin and they also show its asymptotic normality when the initial reserve is zero.

In Subsections 2.1 and 2.2 we suggest a testing problem for the probability of ruin in the infinite time horizon when $F$ is unknown, but a sample from it is available. We then provide a bootstrap P-value to this testing problem, which is asymptotically more accurate than the asymptotic normal P-value.

\subsection{The testing problem}

Let us assume that the probability of ruin can be expressed as the functional

$$
\psi\left(\cdot ; r_{0}\right): \mathcal{F} \rightarrow[0,1], \quad F \mapsto \mathbf{P}_{F}(T<\infty),
$$

where $\mathcal{F}$ denotes the infinite dimensional space of distribution functions on $\mathbb{R}_{+}$with finite expectation, which are the individual claim amount distribution 
functions, and where $\mathbf{P}_{F}$ denotes the probability measure of the underlying filtered probability space that assigns distribution function $F$ to the individual claim amounts. For notational convenience, the initial reserve will serve as a parameter to this functional so that, for any fixed $F \in \mathcal{F}, \psi\left(F ; r_{0}\right)$ becomes a function of $r_{0} \geq 0$. The functional (3) can be defined explicitly as

$$
\psi\left(F ; r_{0}\right)=1-\sum_{k=0}^{\infty} p_{k} F_{L}^{* k}\left(F ; r_{0}\right)
$$

where $p_{k}=\beta(1+\beta)^{-(k+1)}, F_{L}^{* k}$ denotes the $k$-th convolution power of $F_{L}$, for $k \in\{0,1, \ldots\}, \mu(F)=\int_{0}^{\infty} x \mathrm{~d} F(x)$ and

$$
F_{L}(F ; x)=\frac{1}{\mu(F)} \int_{0}^{x}[1-F(y)] \mathrm{d} y
$$

for all $x \geq 0$. The functional representation (3) to the probability of ruin is the one adapted by Pitts (1994, p. 551). We consider $F$ unknown and distinguish the following two situations. In the first one, $F$ belongs to a parametric class, which is a finite dimensional subset of $\mathcal{F}$, with one or more unknown parameters that need to be estimated by the observed claim amounts that occurred within the time interval $[0, t]$, for some $t \in(0, \infty)$. In the second situation, $F \in \mathcal{F}$ is fully unknown and estimated by the empirical distribution function of the observed claim amounts incurred during $[0, t]$. Note that the finite time interval $[0, t]$ is only considered for the estimation of $F$ and that we are still considering probabilities of ruin over the infinite time horizon.

Our parameter of interest is $\psi\left(F ; r_{0}\right)$, where $r_{0}$ is a fixed initial reserve, for which we suggest the testing problem specified by the null and the alternative hypotheses

$$
\begin{aligned}
& \mathrm{H}_{0}: \psi\left(F ; r_{0}\right)=\psi_{0}, \\
& \mathrm{H}_{1}: \psi\left(F ; r_{0}\right)<\psi_{0} .
\end{aligned}
$$

Undervaluing the probability of ruin in an insurance company could lead to inappropriate managerial decisions, for example to an excessive reduction of the capital, which can be dangerous for the company. With the null and alternative hypotheses formulated in (5), the risk of undervaluation of the probability of ruin is controlled by the error of the first kind or the size of the test, which is chosen very small (typically between $1 \%$ and $5 \%$ ). A P-value for this testing problem is given in Subsection 2.2 and approximated with the bootstrap principle.

\subsection{The bootstrap P-value}

As already mentioned, it is often preferable to consider the testing problem (5) instead of a single evaluation of the probability of ruin. In this testing problem, 
it is also more informative to quantify the closeness of the null model to the observed data instead of simply deciding between rejecting the null model or not. The P-value approach leads to this quantification, as a large P-value reflects high coherence of the null model with the data.

Suppose that exactly $n$ claim amounts occur during the time interval $[0, t]$, hence that $N_{t}=n$ for some $t \in(0, \infty)$ and $n \in\{1,2, \ldots\}$. Denote by $\hat{F}_{n}(x)=$ $n^{-1} \sum_{i=1}^{n} 1\left\{X_{i} \leq x\right\}$, for all $x>0$, the empirical distribution function of the claim amounts occurring during $[0, t]$. Consider the functionals

$$
\sigma^{2}\left(F ; r_{0}\right)=\operatorname{var}_{F}\left(\sqrt{n} \psi\left(\hat{F}_{n} ; r_{0}\right)\right),
$$

the variance being taken under $\mathbf{P}_{F}$ and based on the $N_{t}=n$ random claim amounts that occur during $[0, t]$, and

$$
R_{n}(z ; F)=\mathbf{P}_{F}\left(\sqrt{n} \frac{\psi\left(\hat{F}_{n} ; r_{0}\right)-\psi\left(F ; r_{0}\right)}{\sigma\left(\hat{F}_{n} ; r_{0}\right)} \leq z\right)
$$

where $F \in \mathcal{F}$ and $z \in \mathbb{R}$. Denote by $\hat{F}_{n}^{\text {obs }}$ the empirical distribution of $n$ observed claim amounts, i.e. a realization of $\hat{F}_{n}$. Then

$$
P_{n}=\sup _{\left\{F \in \mathcal{F}: \psi\left(F ; r_{0}\right)=\psi_{0}\right\}} R_{n}\left(\sqrt{n} \frac{\psi\left(\hat{F}_{n}^{\text {obs }} ; r_{0}\right)-\psi_{0}}{\sigma\left(\hat{F}_{n}^{\text {obs }} ; r_{0}\right)} ; F\right)
$$

is a P-value for the testing problem (5). Unfortunately, computing the P-value (7) in general involves two major difficulties: the first one is the determination of the distribution of the studentized probability of ruin under all $F \in \mathcal{F}$ such that $\psi\left(F ; r_{0}\right)=\psi_{0}$ and the second one is the evaluation of the supremum over this subset of $\mathcal{F}$. The second difficulty can be reduced by assuming a simple parametric model for the individual claim amounts, which would restrict the search for the supremum from over an infinite dimensional to over a low dimensional space. Nevertheless, in some practical situations it is preferable to avoid this assumption.

As mentioned in the beginning of Section 2, the asymptotic normality of the probability of ruin is established by Pitts (1994, Theorem 5.2). Thus, a first general solution to our problem is to rely on the asymptotic normal approximation

$$
k_{0}(z ; F) \stackrel{\text { def }}{=} \lim _{n \rightarrow \infty} R_{n}(z ; F)=\Phi(z)
$$

for all $z \in \mathbb{R}$ and all $F \in \mathcal{F}$, where $\Phi$ denotes the standard normal distribution function. The independence of the above limit from $F$ is a consequence of the 
(asymptotic) pivotality obtained by studentizing the estimator of the probability of ruin in (6). The asymptotic P-value

$$
P_{A, n}=k_{0}\left(\sqrt{n} \frac{\psi\left(\hat{F}_{n}^{\mathrm{obs}} ; r_{0}\right)-\psi_{0}}{\sigma\left(\hat{F}_{n}^{\mathrm{obs}} ; r_{0}\right)} ; F\right)=\Phi\left(\sqrt{n} \frac{\psi\left(\hat{F}_{n}^{\mathrm{obs}} ; r_{0}\right)-\psi_{0}}{\sigma\left(\hat{F}_{n}^{\text {obs }} ; r_{0}\right)}\right),
$$

for all $F \in \mathcal{F}$, can be evaluated directly, but it is only first-order accurate, in the sense that, for all $F \in \mathcal{F}$,

$$
P_{A, n}-P_{n}=\mathrm{O}\left(n^{-\frac{1}{2}}\right), \text { as } n \rightarrow \infty .
$$

The asymptotic error in (9) follows from expansion (11) below. An asymptotic improvement to (9) can be obtained by applying the bootstrap principle as follows. Consider

$$
R_{n}\left(z ; \hat{F}_{n}\right)=\mathbf{P}_{\hat{F}_{n}}\left(\sqrt{n} \frac{\psi\left(\hat{F}_{n}^{*} ; r_{0}\right)-\psi\left(\hat{F}_{n} ; r_{0}\right)}{\sigma\left(\hat{F}_{n}^{*} ; r_{0}\right)} \leq z\right),
$$

for all $z \in \mathbb{R}$, where $\mathbf{P}_{\hat{F}_{n}}$ is the conditional probability measure where the unknown $F$ has been replaced by $\hat{F}_{n} \in \mathcal{F}$, given the claim amounts $X_{1}, \ldots, X_{n}$ during $[0, t]$, and where $\hat{F}_{n}^{*}$ denotes the empirical distribution function of a random sample generated from $\hat{F}_{n}$, given $X_{1}, \ldots, X_{n}$. From the asymptotic normality of the probability of ruin, we can assume that the expansion

$$
R_{n}(z ; F)=k_{0}(z ; F)+n^{-\frac{1}{2}} k_{1}(z ; F)+\mathrm{o}\left(n^{-\frac{1}{2}}\right), \text { as } n \rightarrow \infty,
$$

holds, thus

$$
R_{n}\left(z ; \hat{F}_{n}\right)=k_{0}\left(z ; \hat{F}_{n}\right)+n^{-\frac{1}{2}} k_{1}\left(z ; \hat{F}_{n}\right)+\mathrm{o}_{\mathrm{P}}\left(n^{-\frac{1}{2}}\right), \text { as } n \rightarrow \infty .
$$

Given two sequences of random variables $\left\{U_{n}\right\}_{n \geq 0}$ and $\left\{V_{n}\right\}_{n \geq 0}$, the notation $U_{n}=\mathrm{o}_{\mathrm{P}}\left(V_{n}\right)$, as $n \rightarrow \infty$, means that $U_{n} / V_{n} \stackrel{\mathrm{P}}{\rightarrow} 0$, as $n \rightarrow \infty$. Expansion (11) is in fact an Edgeworth expansion combined with expansions of the first cumulants of the studentized probability of ruin and $k_{1}(z ; F)$ is the product of a polynomial of degree two in $z$ with the standard normal density at $z$, refer to Hall (1992, Section 2.3) for further explanations. Because $k_{1}\left(z ; \hat{F}_{n}\right)-k_{1}(z ; F)=\mathrm{o}_{\mathrm{P}}(1)$, as $n \rightarrow \infty$, and $k_{0}(z ; F)=\Phi(z)$ is in fact independent of $F$, we have

$$
R_{n}\left(z ; \hat{F}_{n}\right)-R_{n}(z ; F)=\mathrm{o}_{\mathrm{P}}\left(n^{-\frac{1}{2}}\right), \text { as } n \rightarrow \infty,
$$

for all $F \in \mathcal{F}$. Defining 


$$
P_{\mathrm{B}, n}=R_{n}\left(\sqrt{n} \frac{\psi\left(\hat{F}_{n}^{\mathrm{obs}} ; r_{0}\right)-\psi_{0}}{\sigma\left(\hat{F}_{n}^{\mathrm{obs}} ; r_{0}\right)} ; \hat{F}_{n}\right)
$$

approximation (12) yields, for all $F \in \mathcal{F}$,

$$
P_{\mathrm{B}, n}-P_{n}=\mathrm{o}_{\mathrm{P}}\left(n^{-\frac{1}{2}}\right), \quad \text { as } n \rightarrow \infty
$$

whose comparison with (9) shows that the bootstrap $\mathrm{P}$-value $P_{\mathrm{B}, n}$ is asymptotically more accurate than the asymptotic $\mathrm{P}$-value $P_{\mathrm{A}, n} . P_{\mathrm{B}, n}$ is second-order accurate, whereas $P_{\mathrm{A}, n}$ is first-order accurate.

In the remaining part of this section we give some remarks. The P-values $P_{n}, P_{A, n}$ and $P_{B, n}$ are all based on $n$ observed claim amounts, i.e. $n$ realizations from $F$. If we replace these observed claim amounts by their random counterpart, we obtain the random versions of these P-values, denoted by $\tilde{P}_{n}, \tilde{P}_{\mathrm{A}, n}$ and $\tilde{P}_{\mathrm{B}, n}$ respectively. It can be shown that

$$
\mathbf{P}_{F}\left(\tilde{P}_{n} \leq u\right) \leq u
$$

for all $u \in[0,1]$ and all $F \in \mathcal{F}$ such that $\psi\left(F ; r_{0}\right)=\psi_{0}$. This allows for the following error rate interpretation: if we decide to reject $\mathrm{H}_{0}$ whenever a $\mathrm{P}$-value is smaller than or equal to $P_{n}$, then (14) shows that the probability of a false rejection is smaller than or equal to $P_{n}$. For a general reference about P-values, see Casella and Berger (2002, Section 8.3.4).

If we had a simple null hypothesis, the supremum in the definition of $P_{n}$ in (7) would be irrelevant and, as a consequence, the weak inequality outside the probability in (14) would become an equality. In this case, (14) would tell that the P-value is uniformly distributed. But because neither $P_{\mathrm{A}, n}$ nor $P_{\mathrm{B}, n}$ involve the supremum, it follows that both $\tilde{P}_{\mathrm{A}, n}$ and $\tilde{P}_{\mathrm{B}, n}$ are asymptotically uniformly distributed. The uniformity of both $\tilde{P}_{\mathrm{A}, n}$ and $\tilde{P}_{\mathrm{B}, n}$ will be checked in the simulation study of Section 3.

As already mentioned, computing the probability of ruin is generally not a simple problem. We suggest using a numerical approximation introduced by Dufresne and Gerber (1989), which yields upper and lower bounds with arbitrary precision. It is briefly outlined in the Appendix.

For the estimation of the standard error of the estimator of the probability of ruin we use the jackknife. The jackknife is a useful nonparametric technique that allows to estimate various types of quantities and that shares some similarities with the bootstrap, see Quenouille (1949), Tukey (1958), Efron (1979) and Efron (1982). Typical applications are for bias and variance estimation. In our situation, the jackknife estimator of the standard error is given by

$$
\frac{1}{\sqrt{n}} \sigma\left(\hat{F}_{n} ; r_{0}\right) \simeq \sqrt{\frac{n-1}{n} \sum_{i=1}^{n}\left(\psi\left(\hat{F}_{n-1, i} ; r_{0}\right)-\frac{1}{n} \sum_{j=1}^{n} \psi\left(\hat{F}_{n-1, j} ; r_{0}\right)\right)^{2}}
$$


where, for $i \in\{1, \ldots, n\}, \hat{F}_{n-1, i}$ is the $i$-th "leave-one-out" version of $\hat{F}_{n}$, i.e., the empirical distribution function based on the sample $X_{1}, \ldots, X_{i-1}, X_{i+1}, \ldots, X_{n}$.

\subsection{Remarks}

In principle, the results derived in this article can be extended to the probability of ruin within a finite time horizon. In this case, we should first note that the proof of the asymptotic normality of the probability of ruin given by Pitts (1994, Theorem 5.2) is valid in the infinite horizon only. Loisel et al. (2008) prove the asymptotic normality of the finite horizon probability of ruin only in the case where the initial reserve is zero. However, numerical simulations in Loisel et al. (2008, Section 7.1) seem to confirm that the asymptotic normality holds for positive initial reserves as well, and this could be sufficient for extending our bootstrap test. Note also that the algorithm used in Section 3 for computing the probability of ruin and summarized in the Appendix is no longer valid for the finite horizon situation.

Both remarks above remain valid if we were interested in probabilities of ruin, within either the infinite or the finite time horizons, under a general renewal process instead of the Poisson process: we would not have asymptotic normality proofs and we would need an alternative algorithm for the computation of the probabilities of ruin.

\section{Simulation Results}

In this section we present two simulation studies that compare the accuracy of the bootstrap with the asymptotic P-values presented in Subsection 2.2. The programs of the computations made in this section are written in the language $R$, see $\mathrm{R}$ Development Core Team (2008). They can be found in the software section at

http://www.stat.unibe.ch/content/research/publications.

For the first simulation study we consider the nonparametric bootstrap approach. Each sample of claim amounts is generated from an exponential distribution and the bootstrap P-value is computed by resampling from the generated sample. For the second simulation study, we consider the parametric bootstrap approach. Each sample of claim amounts is generated from a log-normal distribution and the bootstrap P-value is computed by generating samples from the log-normal distribution with parameters estimated from the generated sample. The results of both simulations are placed side-by-side in the graphs of Figures 1-4. The left graphs refer to the nonparametric approach and the right ones refer to the parametric approach. In both situations we have considered a two-level simulation design in which we first generate 1000 samples and then, for each sample, we generate 1000 bootstrap samples. Following 

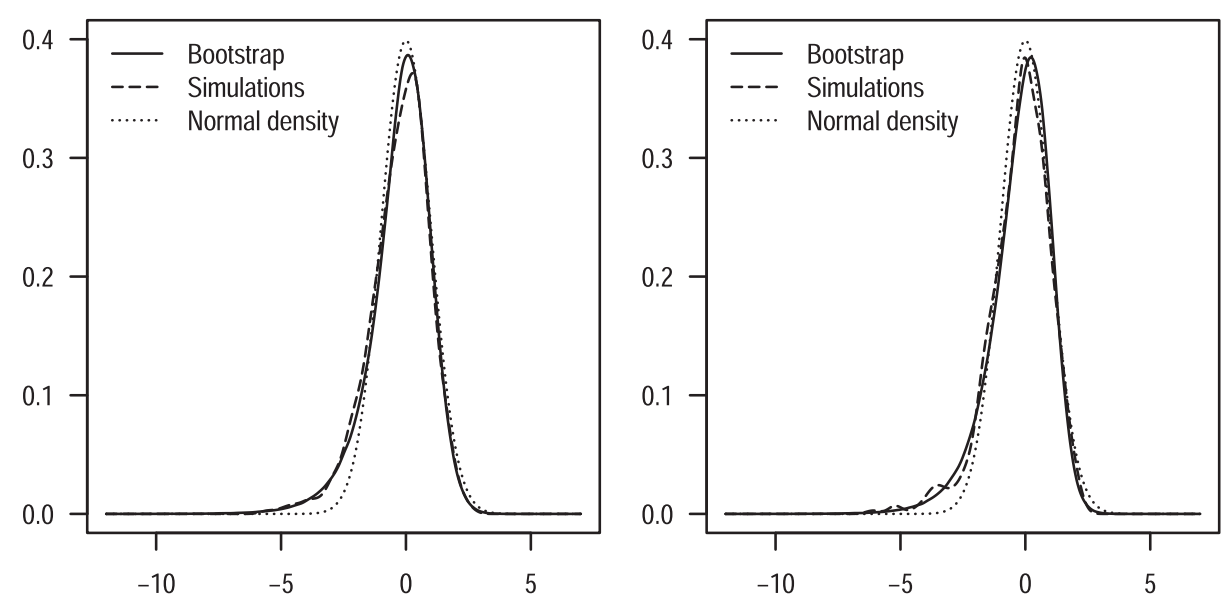

FIGURE 1: Kernel estimators of the density of the studentized probability of ruin based on bootstrap replications (solid lines) and on simulations (dashed lines) with the standard normal density (dotted lines) for exponential (left) and log-normal claims (right).

Hipp (1989a), we assume $\lambda \mu$ (the mean payment per unit of time) known. This assumption avoids difficulties arising whenever the condition $c>\lambda \mu$ is violated by $\lambda$ and $\mu$ replaced by estimated values. Since the parameters $\lambda, \mu$ and $c$ appear in the computation of the probability of ruin only through $\lambda \mu / c=$ $(1+\beta)^{-1}=\psi(F ; 0)$, this assumption reduces to $\beta$ known. For both simulations studies we take $\beta=0.2$ and $\lambda \mu=10$ and thus $\psi(F ; 0)=10 / 12$. Note that the knowledge of $\lambda$ is not necessary in these simulation studies. Practically, there is no need to simulate the Poisson process. We simply generate $n$ claims that are supposed to belong to the time interval $[0, t]$, for some $t \in(0, \infty)$.

In the first simulation study we generate bootstrap P-values when the individual claim amount distribution $F$ is estimated by the empirical distribution of the individual claim amounts. We simulate 1000 samples of $n=250$ individual claim amounts from the exponential distribution with mean 10 . The probability of ruin is computed with the recursive method given in the Appendix, where the distribution function $F_{L}$ defined in (4) is replaced by the estimator given in (18) in the Appendix.

In the second simulation study, bootstrap P-values are based on the parametric estimation of the individual claim amount distribution. We generate 1000 samples of $n=100$ individual claim amounts from the log-normal distribution $F$, where the logarithmic random variable has mean 2 and variance 0.6 . Lower and upper bounds to the probability of ruin are again computed with the recursive algorithm of the Appendix, where the distribution $F_{L}$ defined in (4) is replaced by a parametric estimator of it, by estimating the parameters of $F$ and by numerical integration. In both simulation studies the probabilities of ruin are obtained by taking the average of the upper and the lower bounds defined in (17), using discretization steps of 1 for the estimators of the probability of ruin and of 4 for the estimator of the standard error given in (15). 

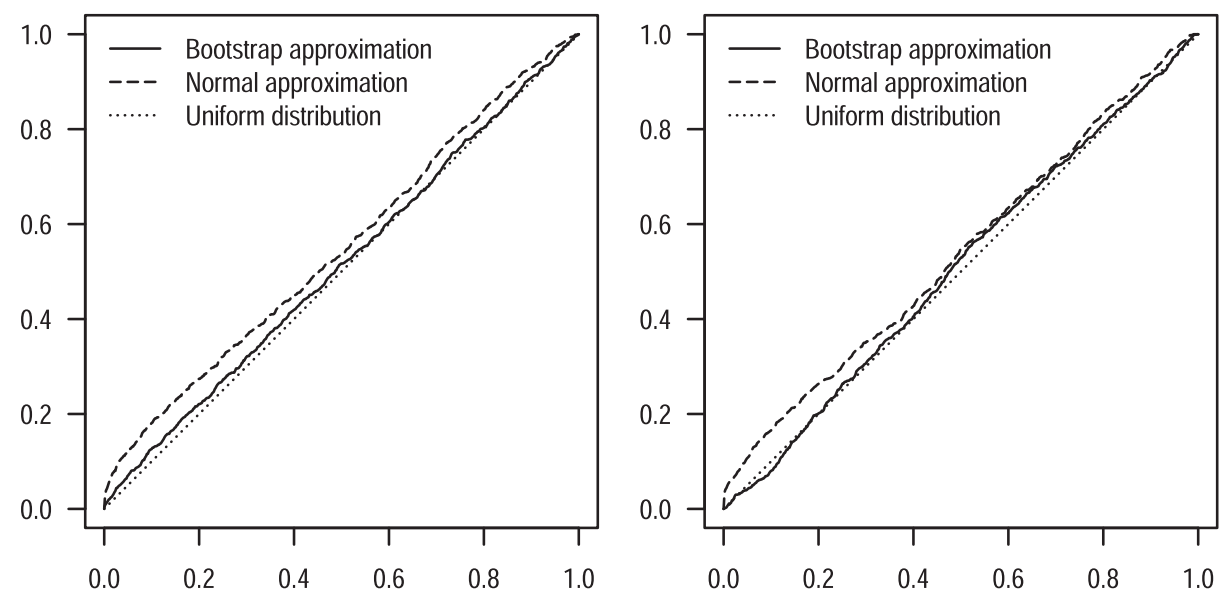

FIGURE 2: Empirical distribution functions of the bootstrap (solid lines) and asymptotic normal (dashed lines) P-values with the uniform distribution function on [0,1] (dotted lines) for exponential (left) and log-normal claims (right).

Figure 1 compares the standard normal density with the kernel density estimators of the studentized probabilities of ruin obtained by 1000 simulations and by $1000 \times 1000$ bootstrap replications. For both the simulated probabilities of ruin and the bootstrap probabilities of ruin we use the kernel density estimator with Gaussian kernels with bandwidths 0.25 and 0.1, respectively. Clearly, the density of the studentized probabilities of ruin is better approximated by the bootstrap densities than by the asymptotic normal densities, which are particularly misleading in the left tails. Figure 2 compares the distribution functions of the bootstrap and asymptotic P-values with the uniform distribution
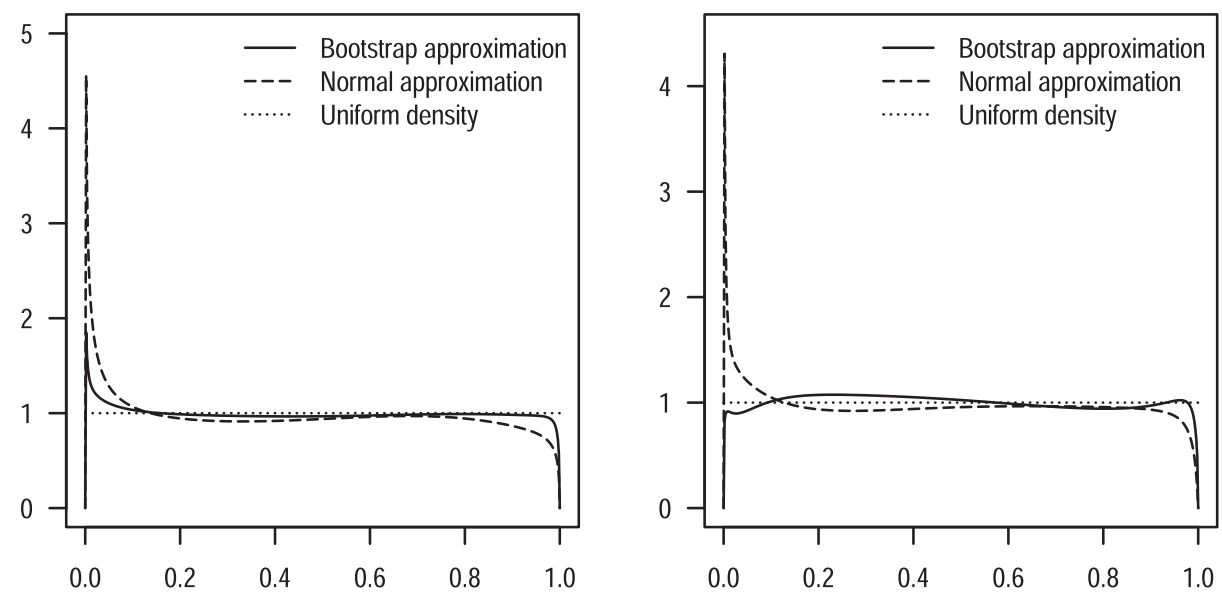

FIGURE 3: Estimated densities of the bootstrap (solid lines) and normal (dashed lines) P-values with the uniform density on $[0,1]$ (dotted lines) for exponential (left) and log-normal claims (right). 

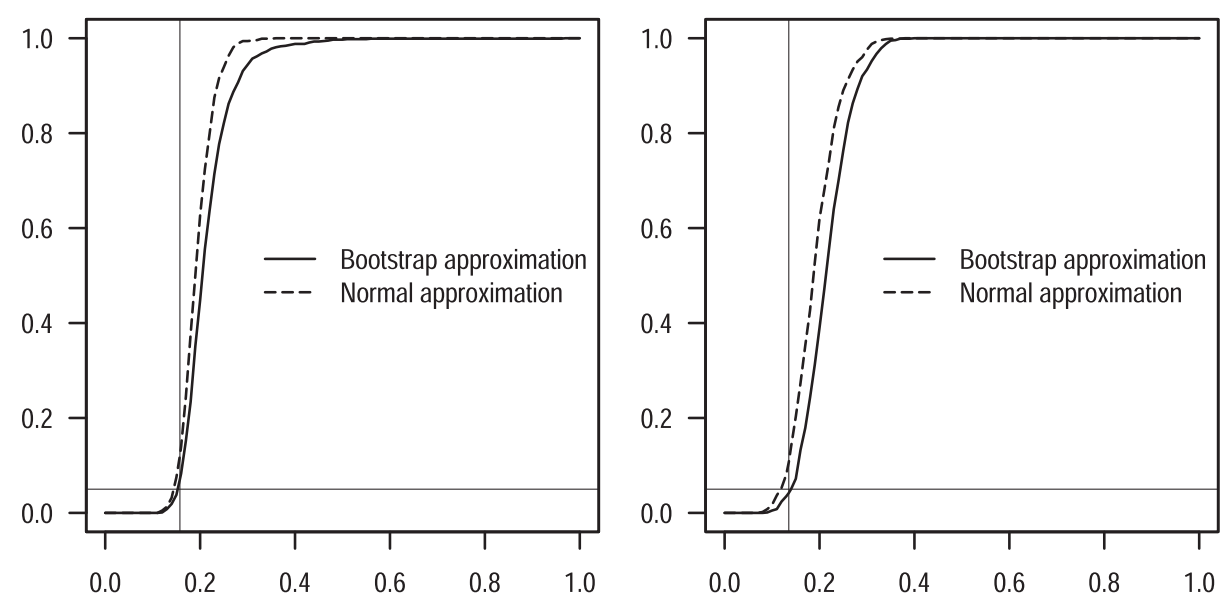

FIGURE 4: Proportion of bootstrap (solid lines) and normal (dashed lines) P-values smaller than or equal to 0.05 as a function of $\psi_{0}$. The horizontal lines indicate the significance level, the vertical lines indicate $\psi\left(F ; r_{0}\right)$ for exponential (left) and log-normal claims (right).

function on $[0,1]$. In both graphs we can see that the bootstrap P-values are closer to uniformity than the asymptotic ones. Thus, bootstrap P-values allow for accurate error rate interpretations, as explained at the end of Subsection 2.2. Asymptotic normal P-values are not close to uniformity and (14) is especially violated for small values, which are the most important ones for the error rate interpretation. Figure 3 shows the densities corresponding to Figure 2. Because the support of the distribution of P-values is bounded, the density estimators of the P-values in Figure 3 use a Gaussian copula-based method with underlying correlation 0.9 instead of the kernel density estimator, see Jones and Henderson (2007) for details. Figure 4 shows how the proportions of P-values smaller than or equal to 0.05 (indicated by the horizontal lines) behave as functions of $\psi_{0}$ with all other parameters left unchanged, for both simulation studies. Because the point $\psi_{0}=\psi\left(F ; r_{0}\right)$ (indicated by the vertical lines) corresponds to the case under which the simulation is performed, it follows from (14) that the proportion at this point should be lower than or equal to 0.05 , which is the level of the test that rejects $\mathrm{H}_{0}$ for P-values smaller than or equal to 0.05 . In both simulation studies, this proportion at this point is close to 0.05 with the bootstrap. The values corresponding to the asymptotic normal approximations exceed the level 0.05 significantly.

\section{ACKNOWLEDGEMENTS}

The authors thank the editor-in-chief, two anonymous referees for their thoughtful comments and the Swiss National Science Foundation for its financial support. 


\section{APPENDIX}

\section{A RECURSIVE METHOD FOR COMPUTING THE PROBABILITY OF RUIN}

In this appendix we summarize an algorithm proposed by Dufresne and Gerber (1989) for computing the probability of ruin within the infinite horizon. Let us define the aggregate loss process $\left\{S_{t}\right\}_{t \geq 0}=\left\{Z_{t}-c t\right\}_{t \geq 0}$ and let $L_{1}, L_{2}, \ldots$ denote the increments of its running maximum $\left\{\max _{0 \leq u \leq t} S_{u}\right\}_{t \geq 0}$, recursively defined by

$$
L_{0}=0 \quad \text { and } \quad L_{k}=S_{\text {inf }\left\{t \geq 0: S_{t}>\sum_{i=0}^{k-1} L_{i}\right\}}-\sum_{i=0}^{k-1} L_{i}
$$

for $k \in\{1,2, \ldots\}$. It can be shown that the maximal aggregate loss $S \stackrel{\text { def }}{=} \max _{t \geq 0}$ $\left\{S_{t}\right\}=L_{1}+\ldots+L_{N}$, where $\mathbf{P}(N=n)=p(1-p)^{n}$, for all $n \in\{0,1, \ldots\}$, where $p=1-\psi(F ; 0)$, and $L_{1}, \ldots, L_{N}$ are independent with the distribution function $F_{L}(F ; x)$, for all $x \geq 0$, defined by (4). The method presented here is essentially based on a discretization of (4) and it leads to upper and lower bounds to $\psi\left(F ; r_{0}\right)$, with both bounds converging to $\psi\left(F ; r_{0}\right)$ with decreasing discretization steps (denoted $\eta$ below).

We can compute a lower and an upper bound for the maximal aggregate loss $S$ by discretizing its summands $L_{1}, \ldots, L_{N}$ on a mesh of width $\eta>0$ as follows,

$$
S^{(\mathrm{L})}=\eta\left\lfloor\eta^{-1} L_{1}\right\rfloor+\cdots+\eta\left\lfloor\eta^{-1} L_{N}\right\rfloor \text { and } S^{(\mathrm{U})}=\eta\left\lceil\eta^{-1} L_{1}\right\rceil+\cdots+\eta\left[\eta^{-1} L_{N}\right\rceil,
$$

where $\lfloor x\rfloor=\max \{k \in \mathbb{Z}: k \leq x\}$ and $\lceil x\rceil=\min \{k \in \mathbb{Z}: k \geq x\}$, for all $x \in \mathbb{R}$. From $\psi\left(F ; r_{0}\right)=\mathbf{P}\left(S>r_{0}\right)$ and $S^{(\mathrm{L})} \leq S \leq S^{(\mathrm{U})}$ it follows that

$$
1-\mathbf{P}\left(S^{(\mathrm{L})}<r_{0}\right) \leq \psi\left(F ; r_{0}\right) \leq 1-\mathbf{P}\left(S^{(\mathrm{U})} \leq r_{0}\right) .
$$

Let us define

$$
h_{k}^{(\mathrm{L})}=\mathbf{P}\left(\eta\left\lfloor\eta^{-1} L_{1}\right\rfloor=\eta k\right)=F_{L}(F ; \eta(k+1))-F_{L}(F ; \eta k),
$$

for $k \in\{0,1, \ldots\}$, and

$$
h_{k}^{(\mathrm{U})}=\mathbf{P}\left(\eta\left\lceil\eta^{-1} L_{1}\right\rceil=\eta k\right)=F_{L}(F ; \eta k)-F_{L}(F ; \eta(k-1)),
$$

for $k \in\{1,2, \ldots\}$. Define also, for $i \in\{0,1, \ldots\}$,

$$
f_{i}^{(\mathrm{L})}=\mathbf{P}\left(S^{(\mathrm{L})}=i \eta\right) \text { and } f_{i}^{(\mathrm{U})}=\mathbf{P}\left(S^{(\mathrm{U})}=i \eta\right) .
$$


From Dufresne and Gerber (1989, Section 2.4), the bounds of (16) can be obtained using the recursive formulae

$$
\begin{aligned}
f_{i}^{(\mathrm{L})} & =\frac{\psi(F ; 0)}{1-\psi(F ; 0) h_{0}^{(\mathrm{L})}} \sum_{k=1}^{i} h_{k}^{(\mathrm{L})} f_{i-k}^{(\mathrm{L})}, \\
f_{i}^{(\mathrm{U})} & =\psi(F ; 0) \sum_{k=1}^{i} h_{k}^{(\mathrm{U})} f_{i-k}^{(\mathrm{U})},
\end{aligned}
$$

for $i \in\{1,2, \ldots\}$, with the initial values

$$
\begin{aligned}
& f_{0}^{(\mathrm{L})}=\frac{1-\psi(F ; 0)}{1-\psi(F ; 0) h_{0}^{(\mathrm{L})}}, \\
& f_{0}^{(\mathrm{U})}=1-\psi(F ; 0) .
\end{aligned}
$$

Note that $\psi(F ; 0)=\lambda \mu / c$ depends on $F$ through its mean only. Precisely, using (16), these bounds are given by

$$
1-\sum_{i=0}^{\left[\eta^{-1} r_{0} \mid-1\right.} f_{i}^{(\mathrm{L})} \leq \psi\left(F ; r_{0}\right) \leq 1-\sum_{i=0}^{\left\lfloor\eta^{-1} r_{0}\right\rfloor} f_{i}^{(\mathrm{U})}
$$

In the first example of Section 3, $F$ is unknown and estimated by the empirical distribution function $\hat{F}_{n}$ of the claim amounts. In this case we estimate $F_{L}$ by

$$
\begin{aligned}
F_{L}\left(\hat{F}_{n} ; x\right) & =\frac{1}{\mu\left(\hat{F}_{n}\right)} \int_{0}^{x}\left[1-\hat{F}_{n}(y)\right] \mathrm{d} y \\
& =\frac{1}{\mu\left(\hat{F}_{n}\right)} \int_{0}^{x} \frac{1}{n} \sum_{i=1}^{n} \mathbf{1}\left\{y<X_{i}\right\} \mathrm{d} y \\
& =\frac{\sum_{i=1}^{n} \min \left(x, X_{i}\right)}{\sum_{i=1}^{n} X_{i}}
\end{aligned}
$$

where $n$ is the number of claims observed during $[0, t]$.

\section{REFERENCES}

Asmussen, S. (1985) Conjugate processes and the simulation of ruin problems, Stochastic Processes and their Applications, 20, 213-229.

Asmussen, S. (2000) Ruin Probabilities, vol. 2 of Advanced Series on Statistical Science and Applied Probability, World Scientific, Singapore. 
Asmussen, S. and Rolski, T. (1991) Computational methods in risk theory: A matrix-algorithmic approach, Insurance: Mathematics and Economics, 10, 259-274.

BARNDORFF-NiElSEN, O.E. and SChMidli, H.U. (1995) Saddlepoint approximations for the probability of ruin in finite time, Scandinavian Actuarial Journal, 2, 169-186.

Bowers, N., Gerber, H.U., Hickmann, J., Jones, D. and Nesbitt, C. (1997) Actuarial Mathematics, The Society of Actuaries, Schaumburg, Illinois, second edn.

Casella, G. and Berger, R.L. (2002) Statistical Inference, Duxbury Advanced Series in Statistics and Decision Sciences, Duxbury Press, Pacifice Grove, California, second edn.

Davison, A.C. and Hinkley, D.V. (2003) Bootstrap Methods and their Applications, no. 1 in Cambridge Series in Statistical and Probabilistic Mathematics, Cambridge University Press, Cambridge, reprinted and corrected edn.

Dufresne, F. and Gerber, H.U. (1989) Three methods to calculate the probability of ruin, ASTIN Bulletin, 19, 71-90.

EFron, B. (1979) Bootstrap methods: Another look at the jackknife, The Annals of Statistics, 7, $1-26$.

EFron, B. (1982) The Jackknife, the Bootstrap and Other Resampling Plans, no. 38 in CBMS-NSF Regional Conference Series in Applied Mathematics, Society for Industrial and Applied Mathematics (SIAM), Philadelphia.

Embrechts, P. and Miкosch, T. (1991) A bootstrap procedure for estimating the adjustment coefficient, Insurance: Mathematics and Economics, 10, 181-190.

GATTO, R. (2008) A saddlepoint approximation to the probability of ruin in the compound Poisson process with diffusion, Statistics and Probability Letters, 78, 1948-1954.

Gerber, H.U. (1979) An Introduction to Mathematical Risk Theory, no. 8 in Huebner Foundation Monograph Series, S. S. Huebner Foundation for Insurance Education, Philadelphia.

Hall, P. (1992) The Bootstrap and Edgeworth Expansion, Springer Series in Statistics, SpringerVerlag, New York.

HIPP, C. (1989a) Estimators and bootstrap confidence intervals for ruin probabilities, ASTIN Bulletin, 19, 57-70.

Jones, M.C. and Henderson, D.A. (2007) Kernel-type density estimation on the unit interval, Biometrika, 94, 977-984.

Loisel, S., Mazza, C. and Rullière, D. (2008) Robustness analysis and convergence of empirical finite-time ruin probabilities and estimation risk solvency margin, Insurance: Mathematics and Economics, 42, 746-762.

PitTs, S.M. (1994) Non-parametric estimation of compound distributions with applications in insurance, Annals of the Institute of Statistical Mathematics, 46, 537-555.

Pitts, S.M., GrüBel, R. and Embrechts, P. (1996) Confidence bounds for the adjustment coefficient, Advances in Applied Probability, 28, 802-827.

Politis, K. (2003) Semiparametric estimation for non-ruin probabilities, Scandinavian Actuarial Journal, 1, 75-96.

Quenouille, M.H. (1949) Approximate tests of correlation in time-series, Journal of the Royal Statistical Society. Series B (Methodological), 11, 68-84.

R Development Core Team (2008) R: A Language and Environment for Statistical Computing, $\mathrm{R}$ Foundation for Statistical Computing, Vienna, Austria.

Tukey, J.W. (1958) Bias and confidence in not quite large samples (abstract), The Annals of Mathematical Statistics, 29, 614.

\author{
BENJAMIN BAUMgARTNER and RicCARDo GATTO \\ Institute of Mathematical Statistics and Actuarial Science \\ University of Bern \\ Alpeneggstrasse 22 \\ 3012 Bern \\ Switzerland \\ E-mail: baumgartner@stat.unibe.ch \\ E-mail: gatto@stat.unibe.ch
}

